\title{
Advanced Stirling Convertor Testing at NASA Glenn Research Center
}

\author{
5th International Energy Conversion Engineering Conference
}

\author{
June 25, 2007 \\ Salvatore Oriti \\ NASA Glenn Research Center \\ RPT - Thermal Energy Conversion Branch
}

\begin{abstract}
The U.S. Department of Energy (DOE), Lockheed Martin Space Systems (LMSS), Sunpower Inc., and NASA Glenn Research Center (GRC) have been developing an Advanced Stirling Radioisotope Generator (ASRG) for use as a power system on space science and exploration missions. This generator will make use of the free-piston Stirling convertors to achieve higher conversion efficiency than currently available alternatives. The ASRG will utilize two Advanced Stirling Convertors (ASC) to convert thermal energy from a radioisotope heat source to electricity. NASA GRC has initiated several experiments to demonstrate the functionality of the ASC, including: in-air extended operation, thermal vacuum extended operation, and ASRG simulation for mobile applications. The in-air and thermal vacuum test articles are intended to provide convertor performance data over an extended operating time. These test articles mimic some features of the ASRG without the requirement of low system mass. Operation in thermal vacuum adds the element of simulating deep space. This test article is being used to gather convertor performance and thermal data in a relevant environment. The ASRG simulator was designed to incorporate a minimum amount of support equipment, allowing integration onto devices powered directly by the convertors, such as a rover. This paper discusses the design, fabrication, and implementation of these experiments.
\end{abstract}




\title{
Advanced Stirling Convertor Testing at NASA Glenn Research Center
}

\author{
$5^{\text {th }}$ International Energy Conversion \\ Engineering Conference \\ June 25, 2007 \\ Salvatore Oriti \\ NASA Glenn Research Center \\ RPT - Thermal Energy Conversion Branch
}

Glenn Research Center 


\section{Advanced Stirling Radioisotope Generator}

ENGINEERING UNIT PERFORMANCE

- Power: $\quad$ BOM -140 We

- Mass: $\quad 20.8 \mathrm{~kg}$

- Envelope: $30.5 \mathrm{~cm} \mathrm{~W}, 46 \mathrm{~cm} \mathrm{H}, 76 \mathrm{~cm} \mathrm{~L}$ (12"W, 18"H, 30"L)

- Specific Power:

- Efficiency:

$6.7 \mathrm{We} / \mathrm{kg}$

- Design Life: $28 \%$

14 years plus 3 years storage

ATTRIBUTES

- Two Stirling convertors

- Co-axially aligned for dynamic balance

- One GPHS module per convertor

- Designed to operate in the vacuum of deep space or Martian atmosphere with unimpeded view factor

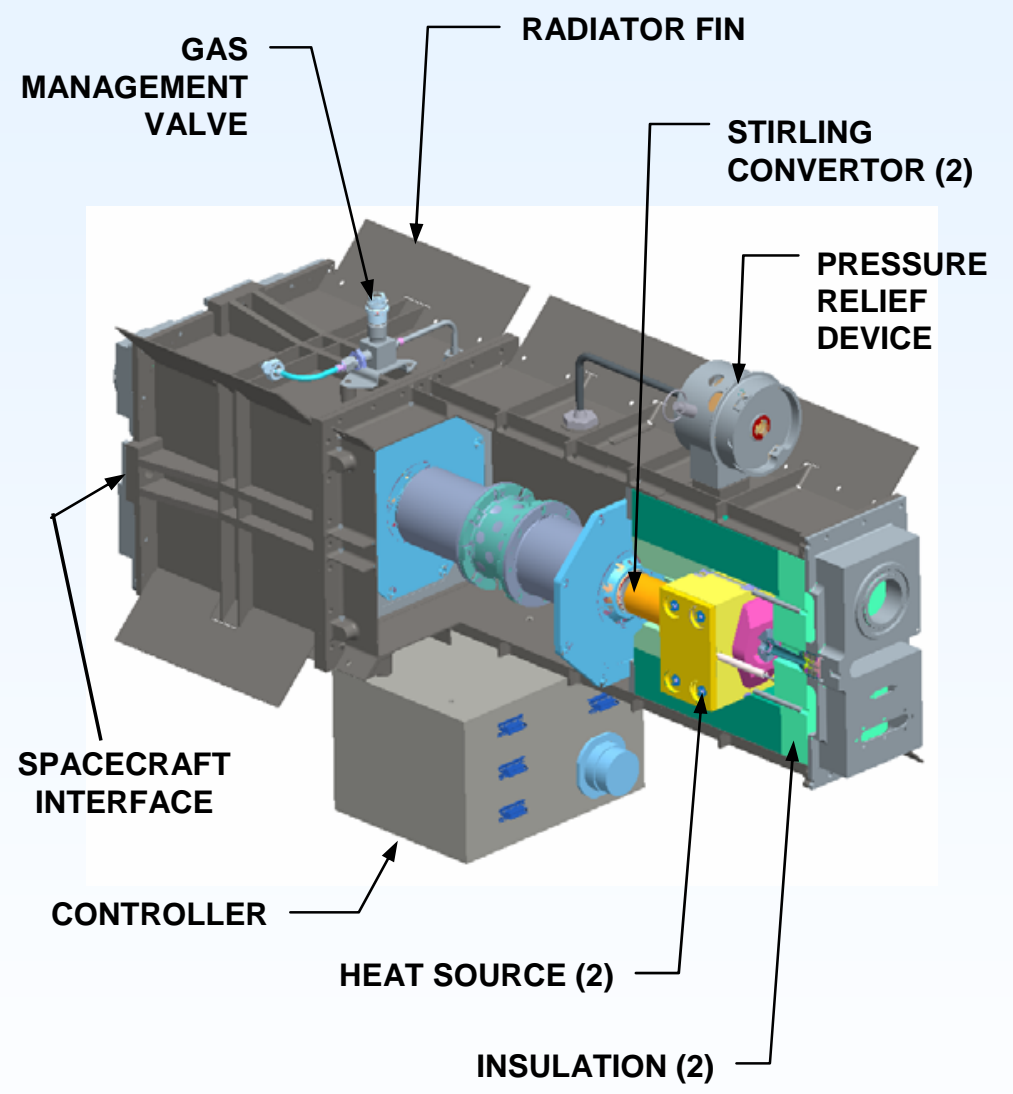

ASRG Engineering Unit

Courtesy of Lockheed Martin Space

Systems

Glenn Research Center 


\section{Advanced Stirling Convertor Testing}

PURPOSES:

1. Demonstrate life and reliability of the convertors

2. Independent validation and verification

3. Track performance over several thousands of hours to evaluate presence of degradation

ASC prototypes available for testing

\begin{tabular}{|c|c|c|c|c|}
\hline Convertor Model & Heater Head Material & Hermetic & Hot-end Temperature, ${ }^{\circ} \mathbf{C}$ & Operation \\
\hline ASC-0 & Inconel'M 718 & Y & 650 & Extended \\
\hline ASC-1HS & MarM-247 & Y & 850 & Extended \\
\hline FTB & Stainless Steel & N & 550 & ASRG simulator \\
\hline
\end{tabular}

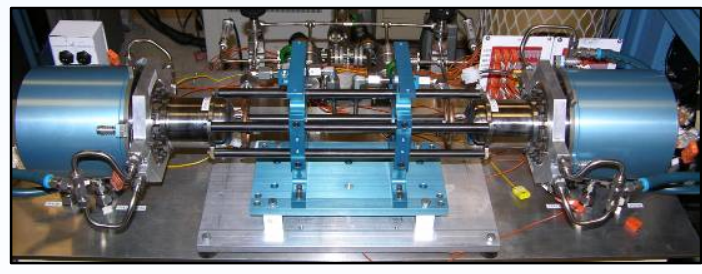

Extended operation in air Pumped loop cooling

Glenn Research Center

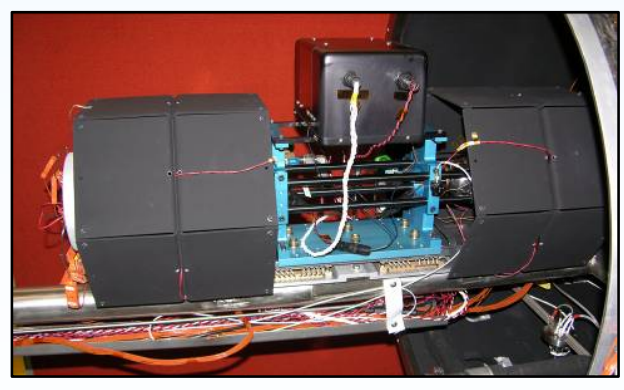

Extended operation in thermal vacuum

Radiation cooling

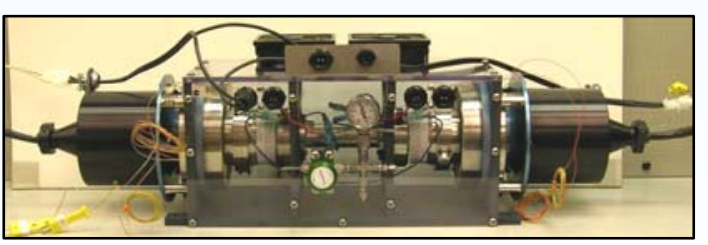

ASRG Simulator

Air cooling 


\section{Extended Operation Test Stations}

\section{Features:}

- Automatic control of support systems

- Operation safely shutdown when potentially dangerous condition exists

- Shutdowns triggered by: hot-end and rejection temperatures, charge pressure, piston amplitude, and loss of building power

- Permits unattended, 24-7 operation to accumulate operation at quickest pace.

- Hard-wired protections:

- Temperature limit controller - removes heater power if excessive hot-end temperature is detected

- Failsafe protection circuit - quickly provides emergency load across alternators if excessive piston amplitude is detected

- Hot-end temperatures control by PID loop and programmable DC power supplies

- $\quad$ Automatic recording of operation data:

- Temperatures, voltage, current, power output, charge pressure

- Gas management system

- Evacuation and backfilling of convertors for ultra high purity charge

- Charge pressure adjustment

- Sampling and analysis of helium charge through residual gas analyzer

- Eight convertors may be operated simultaneously

- 3 pairs in air

- 1 pair in thermal vacuum

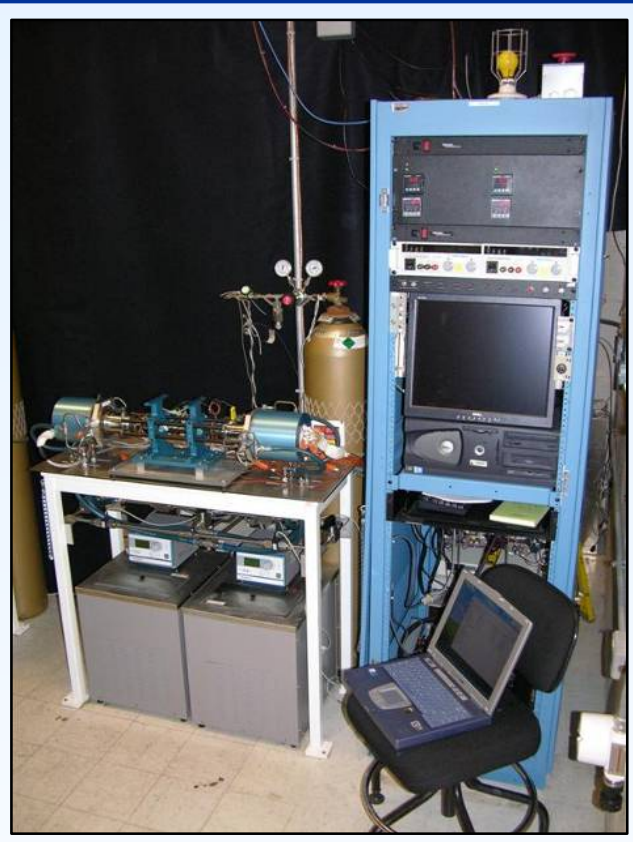

Extended Operation Test Station

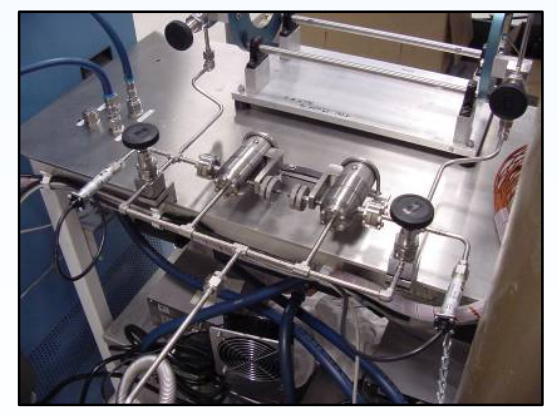

Gas Management Manifold

\section{Glenn Research Center}




\section{In-Air Test Article}

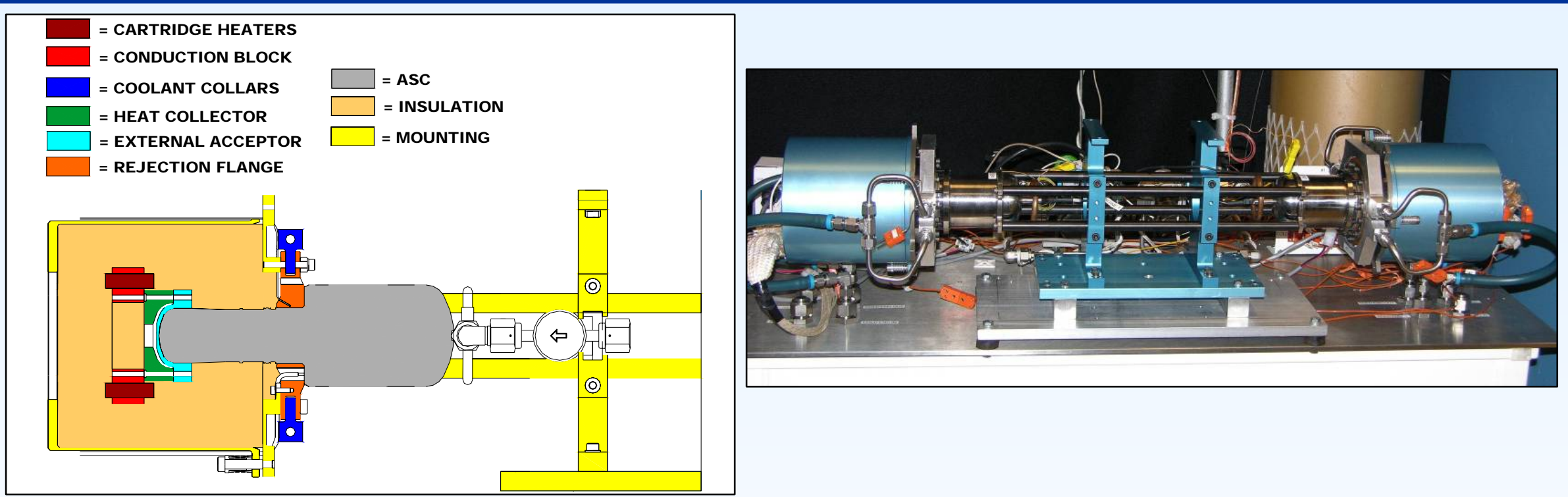

- Two convertors in dual-opposed configuration - hot-ends outward and alternators facing towards center

- Heat rejection:

- Copper flange brazed to rejection zone of heater head

- Aluminum collars with fluid passages

- Temperature controlled fluid provided by laboratory circulator

- Heat input:

- Array of cartridge heaters inserted into hexagonal copper block

- Preloaded onto heat collector by threaded rods

- Insulated with ceramic blanket

Glenn Research Center 


\section{In-Air Hardware Design}

\section{Rejection flange:}

- Designed for in-air and thermal vacuum operation

- Accepts pumped loop collars and radiator panels

- Brazed to ASC heater head during fabrication

- $\quad$ Required to conduct $145 \mathrm{~W}_{\text {th }}$ at $10{ }^{\circ} \mathrm{C}$ temperature drop

- Final geometry predicted to have temperature drop of $6.2^{\circ} \mathrm{C}$ at $145 \mathrm{~W}_{\text {th }}$.

- Material : Copper for high thermal conductivity and machinability

\section{Coolant collars:}

- Occupy minimum space while permitting sufficient coolant flowrate to remove cycle waste heat

- $\quad$ Passage analyzed as straight duct with underdeveloped laminar flow

- Predicted marginal performance

- Measured performance on operating convertors was more than sufficient.

\section{Heat Collector:}

- Designed for in-air and thermal vacuum operation

- Interfaces to cartridge heater block and Boralectric ${ }^{\mathrm{TM}}$ sources

- Material : Nickel 201 for high thermal conductivity and high temperature capability.

- Emulates flat heat source interface as in ASRG

- $\quad$ Designed to minimize temperature drop and survive heater preload at full operating temperature while conducting $220 \mathrm{~W}_{\text {th }}$

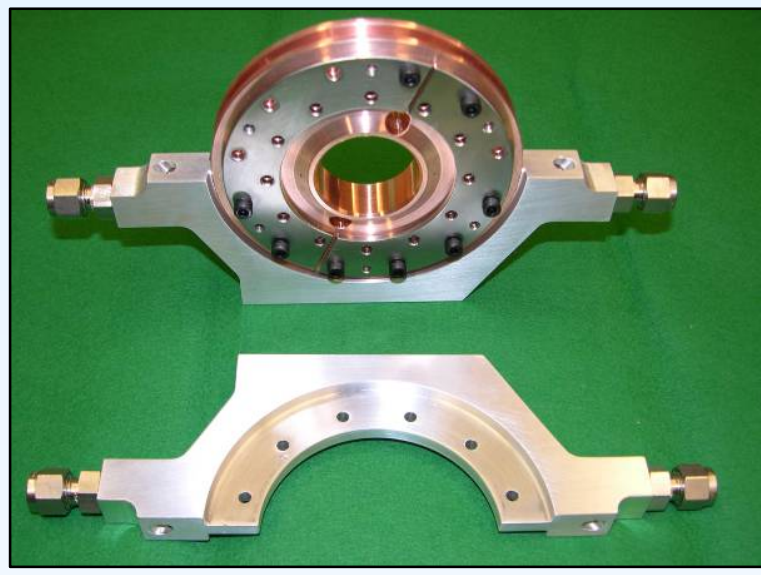

Glenn Research Center

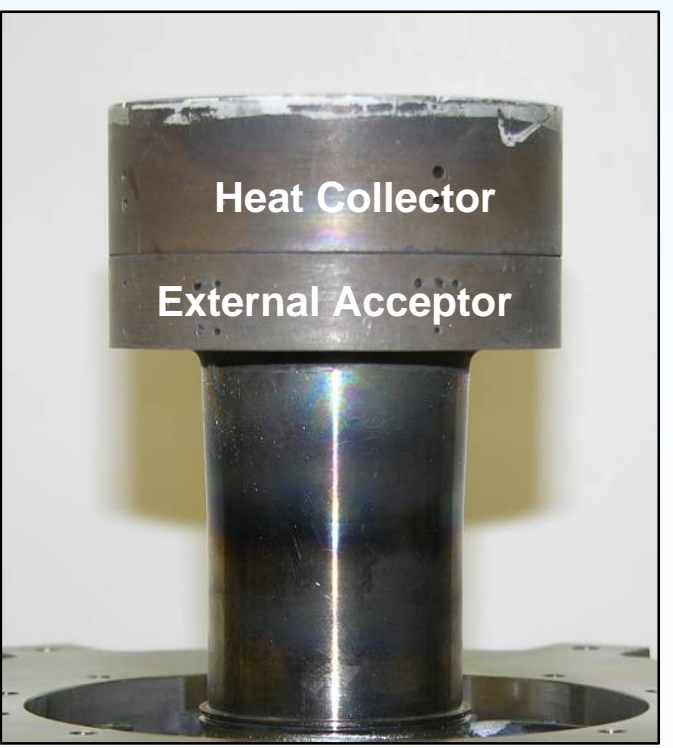




\section{In-Air Operation Summary}

ASC-0 \#1 and \#2

- First pair of hermetically sealed convertors

- Inconel 718 heater heads

- $650{ }^{\circ} \mathrm{C}$ hot-end, $90{ }^{\circ} \mathrm{C}$ rejection

Key dates:

- December 20, 2006 : Received

- February 2, 2007 : Initial operation

- February 8,2007 : Began extended operation :

- March 5, 2007 : Began transition to thermal vacuum operation

Over 600 hours of in-air operation at full design operating conditions

- No failures

- No loss of charge pressure : indicates successful hermetic sealing

- Post processing performance and helium sampling data

- All support hardware performed within design margins

- Rejection hardware capable of maintaining rejection zone at desired temperature at full power

Glenn Research Center 


\section{Thermal Vacuum Test Article}
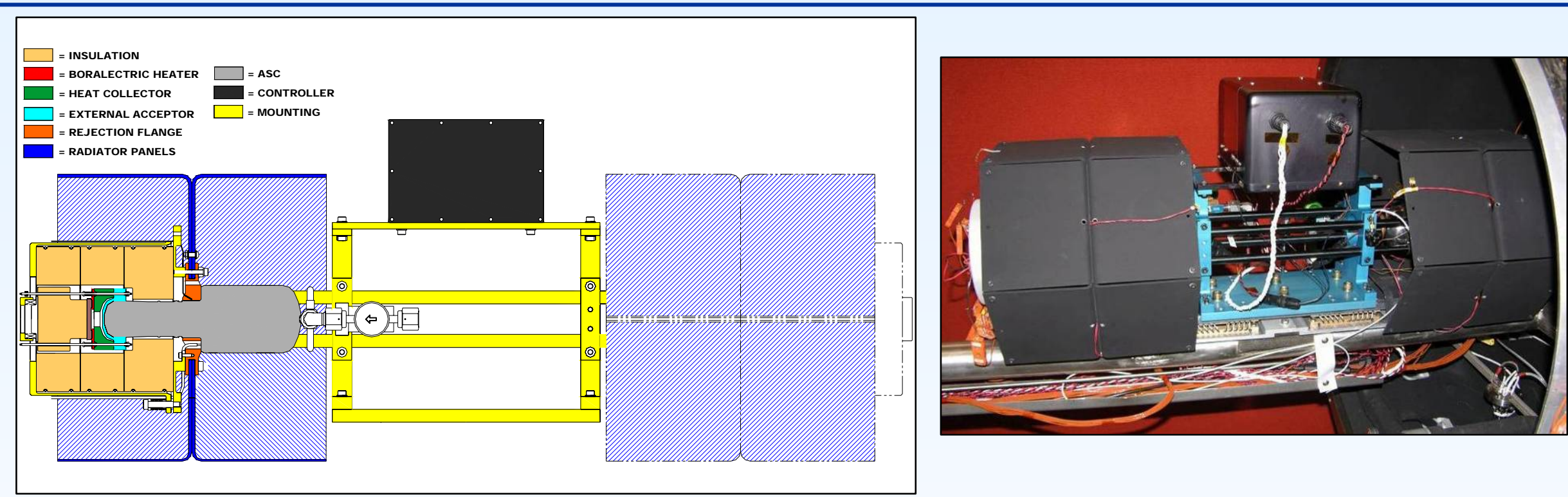

- Two convertors in dual-opposed configuration - hot-ends outward and alternators facing towards center

- Zener-diode controller integrated into center mounting structure (parasitic load outside vacuum)

- Liquid nitrogen-cooled shroud surrounds $100 \%$ of test article

- Heat rejection:

- Aluminum radiator panels coated with ECP-2200 (increases emissivity to 0.9)

- T-gon 805 at copper-aluminum interfaces to reduce thermal contact resistance

- Heat input:

- Compact Boralectric ${ }^{\mathrm{TM}}$ heater

- Preloaded onto collector by backing plate and threaded rod

- Insulated with rigid microporous insulation assembly

Glenn Research Center 


\section{Thermal Vacuum Hardware Design}

\section{Radiator Panels:}

- $\quad$ Designed using system level thermal FEA model

- Included heat load from each convertor $\left(145 \mathrm{~W}_{\mathrm{th}}\right)$, insulation containers, pressure vessels, and controller.

- Required to maintain $90{ }^{\circ} \mathrm{C}$ rejection temperature in this environment

- Liquid nitrogen shroud modeled as fixed radiation sink temperature.

\section{Boralectric $^{\mathrm{TM}}$ heater:}

- Design governed by heat collector geometry and manufacturing limits

- Shape driven to maximum inner and outer diameters to supply heat along path of lowest thermal resistance.

- Fastening hardware made of MarM-246 - similar to MarM-247

- Molybdenum spacers inserted into each fastened joint to eliminate thermally induced stresses

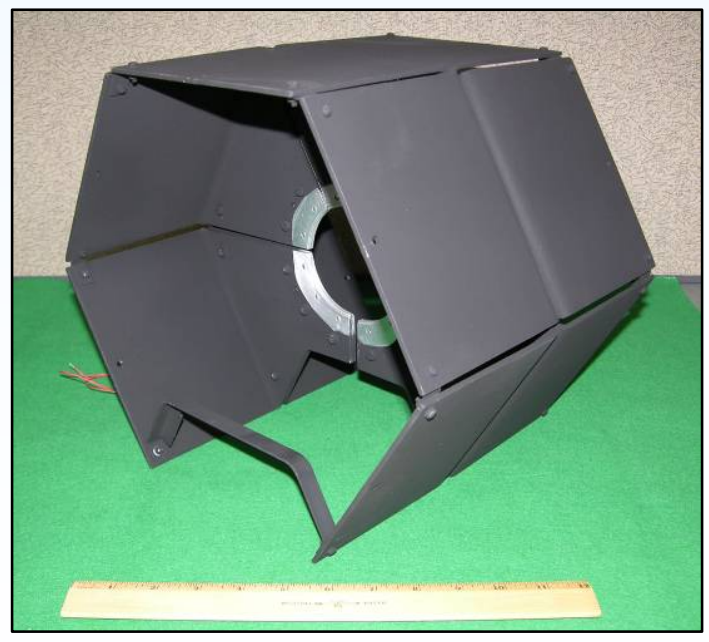

Radiator panel assembly Glenn Research Center

Boralectric heater 


\section{Thermal Vacuum Hardware Design}

- To drive $220 \mathrm{~W}_{\text {th }}$ to heater head at $650{ }^{\circ} \mathrm{C}$, heater will operate at about $720^{\circ} \mathrm{C}$.

- Molybdenum spacers sized to nullify relatively large CTE of heater

- Electric power provided to heater by smaller diameter threaded rods - length extended to protrude through insulation

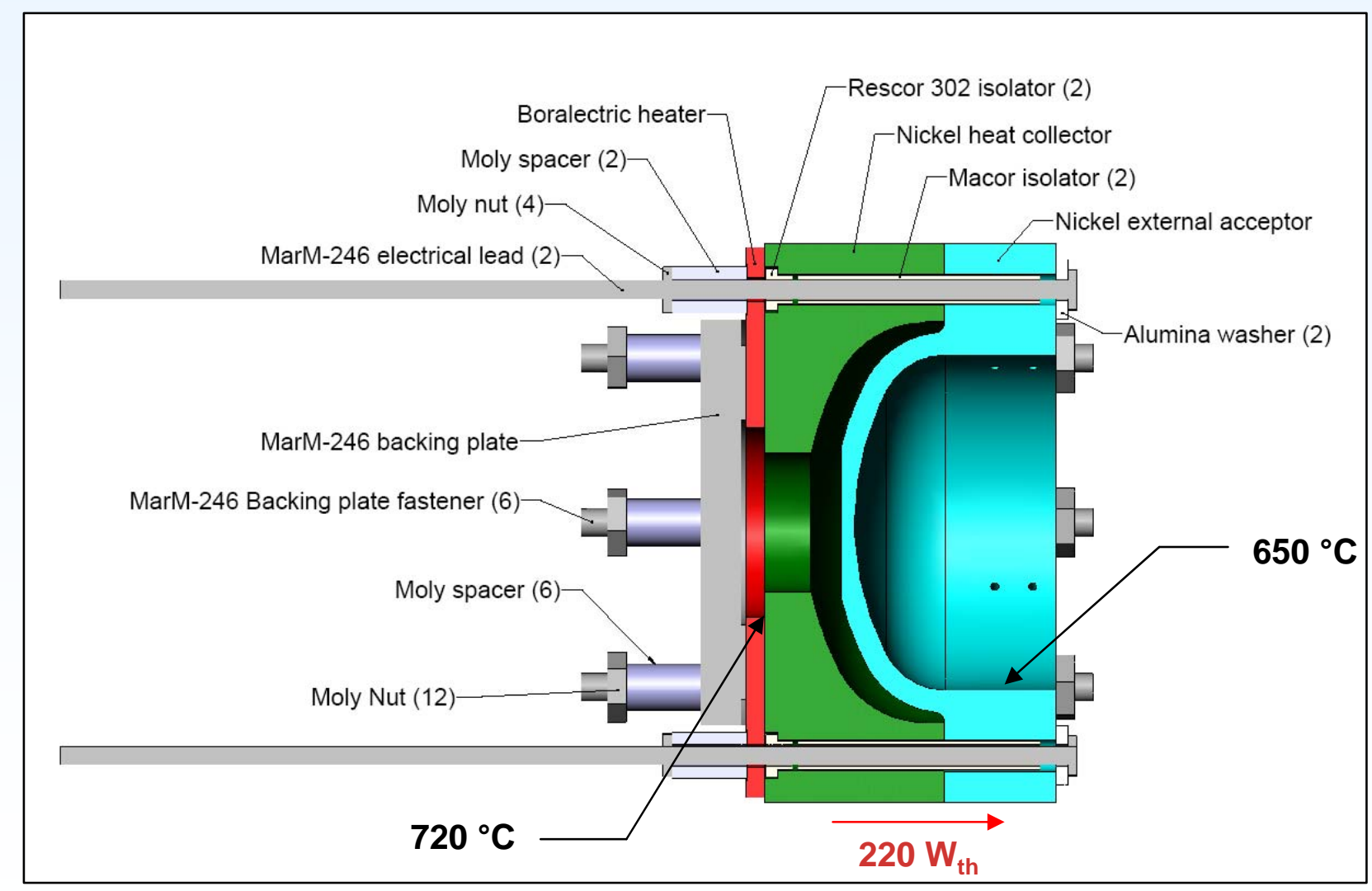

Glenn Research Center

at Lewis Field 


\section{Thermal Vacuum Operation Summary}

ASC-0 \#1 and \#2

- March 5, 2007 : Began transition to thermal vacuum operation

- March 30, 2007 : Initial thermal vacuum checkout

- April 3, 2007 : Began extended operation in thermal vacuum

Over 1600 hours of thermal vacuum operation as of June 25, 2007

Over 800 hours of thermal vacuum operation at maximum hot-end temperature $\left(\sim 650^{\circ} \mathrm{C}\right)$

- No failures

- No loss of charge pressure after repairing leak in external helium management manifold : indicates successful hermetic sealing

- Post processing performance and helium sampling data

- All support hardware performing within design margins

- Radiator panels maintain rejection temperature below $90^{\circ} \mathrm{C}$ at full power

- Boralectric heater assembly has survived $720{ }^{\circ} \mathrm{C}$ operation with no indication of degradation

\section{Future Plans}

- Summer 2007 : Receive ASC-1HS pair and begin thermal vacuum operation of ASC-1HS at hot-end temperature of $850{ }^{\circ} \mathrm{C}$

- Continue extended operation of ASC-0 \#1 and \#2 in air

Total ASC-0 \#1 and \#2 operation at GRC = 2,225 hours as of June 25, 2007

Glenn Research Center 


\section{ASRG Simulator}

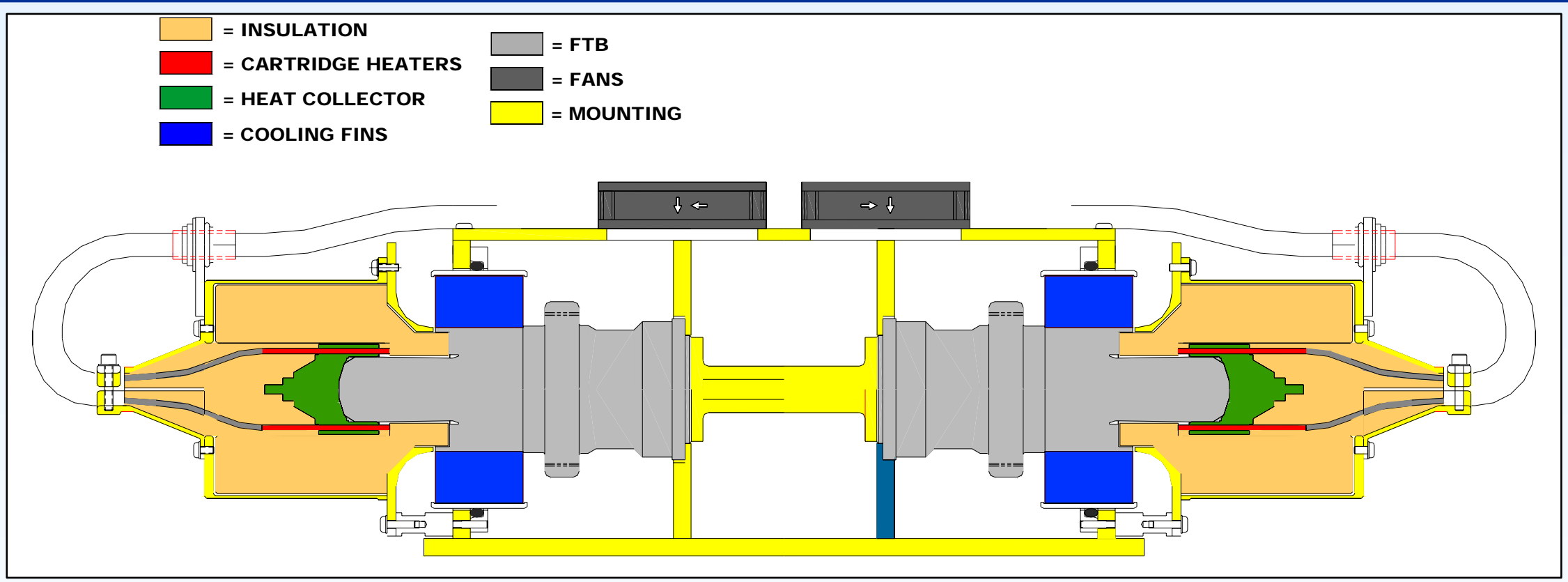

Purpose : Portable system for short-term demonstrations of Stirling energy conversion outside laboratory, such as powering a rover.

- Two FTB convertors in dual-opposed configuration

- Cooling fins soldered onto rejection zone

- Cooling air provided by two fans

- Energy input through cartridge heaters, insulated with ceramic blanket

- Helium manifold for charge pressure adjustment

- Minimum amount of required equipment for operation

Glenn Research Center 


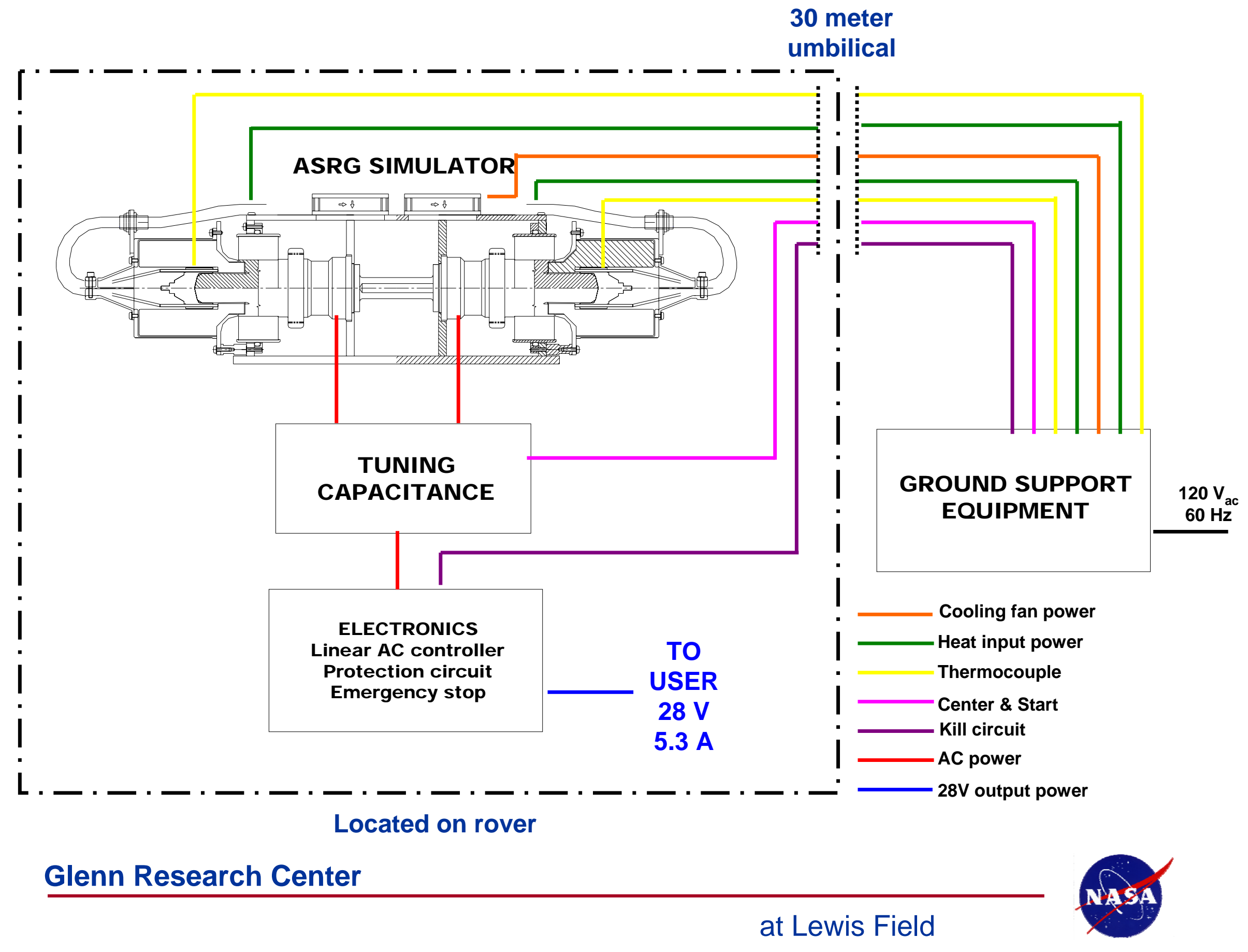




\section{ASRG Simulator Operation Summary}

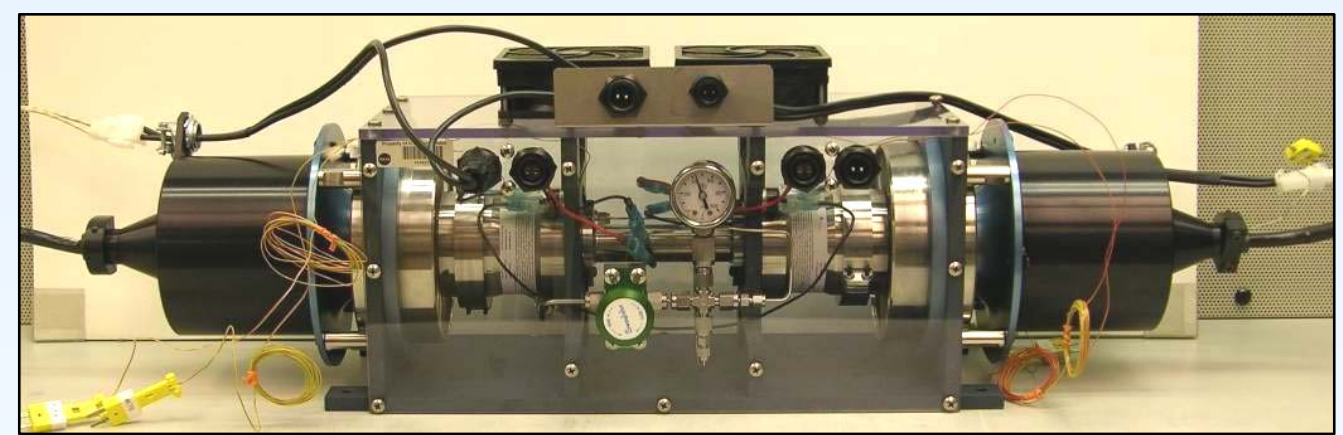

ASRG Simulator

- December 2006 : Buildup complete

- December 2006 : Full power checkout completed up to $130 \mathrm{~W}_{\mathrm{e}}$

Future Plans

- Implement autonomous operation

- Automatic startup and shutdown

- Single user start button

- Summer 2007 : Demonstration as rover power source

Glenn Research Center

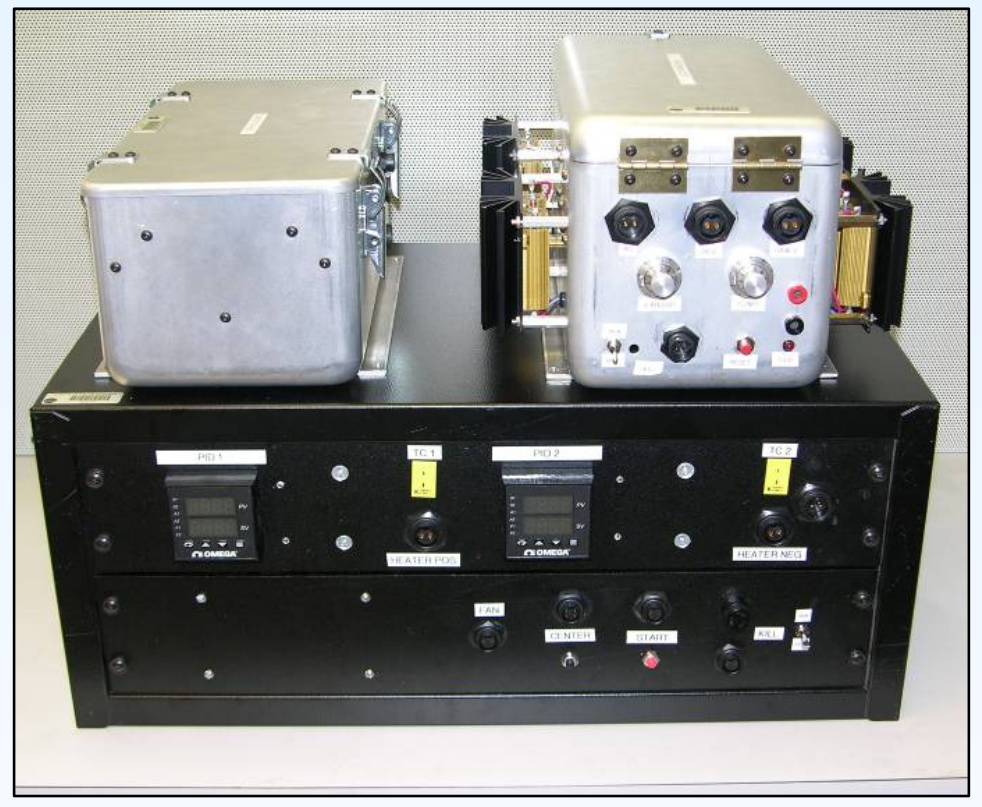

Support equipment 


\section{ASRG Simulator Controller Development}

\section{PURPOSE}

Design and build small efficient, reliable controller for ASRG Simulator operation

- $\quad$ Controller is necessary to dissipate power produced by convertor but not absorbed by user.

- $\quad$ Provides regulated output voltage (usually $28 \mathrm{~V}_{\mathrm{DC}}$ )

- Allows adjustment of piston amplitude

- Six methods evaluated

- $\quad$ Passive power factor correction using tuning capacitors

- $\quad$ Analyzed with PSpice ${ }^{T M}$ and model of ASC alternator pair

1. Zener-diode (baseline)

2. Linear DC regulator

3. Digital hybrid

4. Buck circuit with zener-diode

5. Boost circuit with pulse width modulator

6. Linear $\mathrm{AC}$ regulator 


\section{Zener-diode (baseline)}

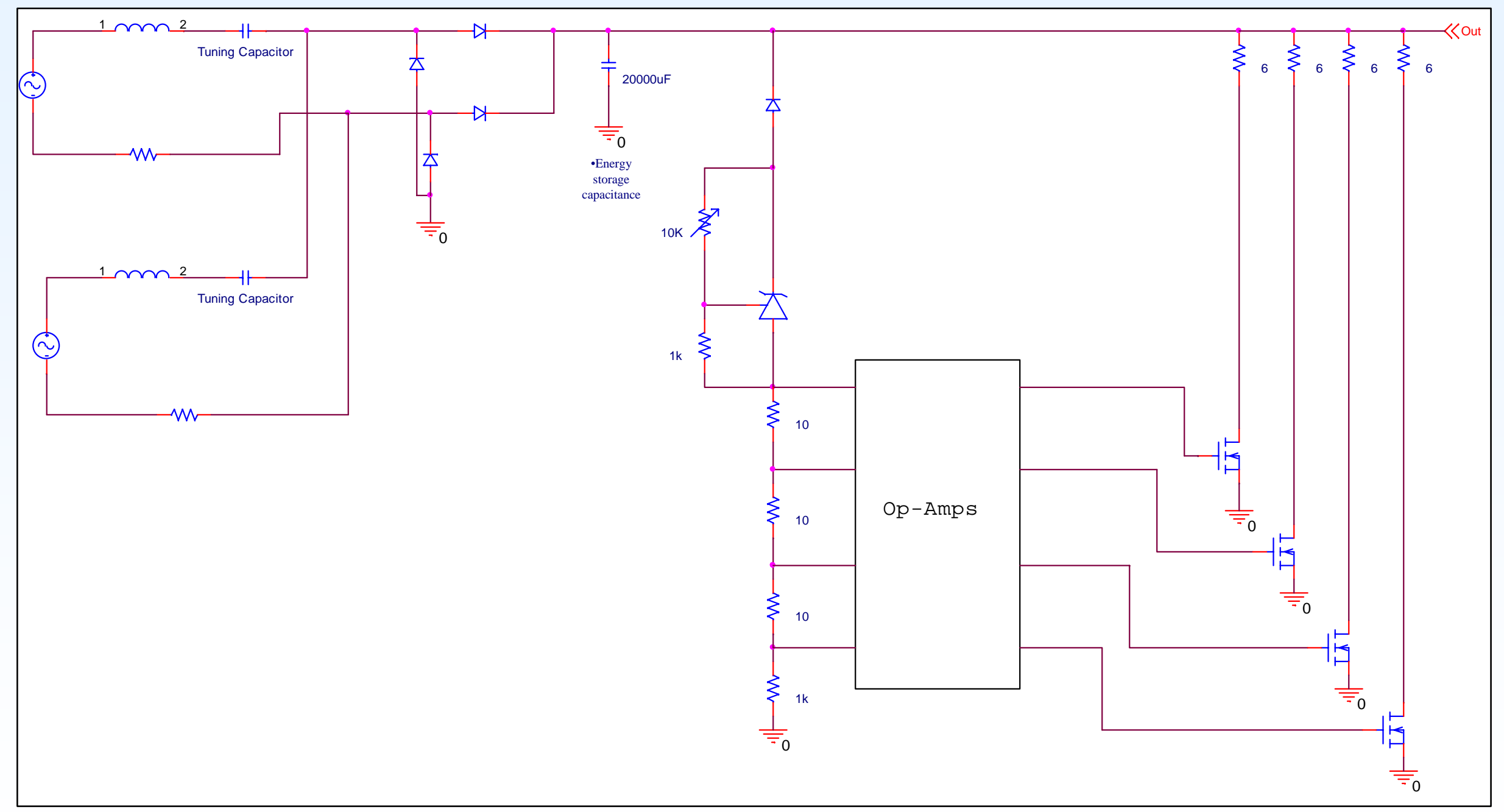

Glenn Research Center

at Lewis Field 


\section{Linear DC Regulator}

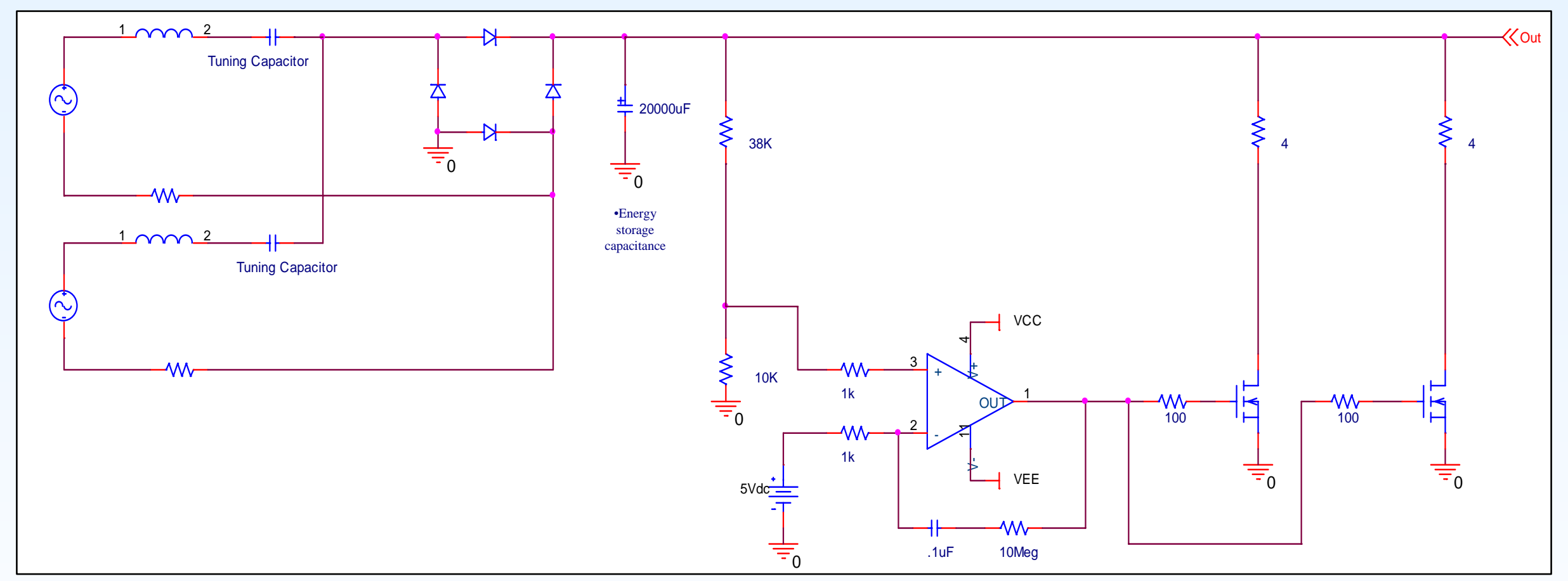

\section{Key Features}

- FETs driven in linear range - also dissipate power

- Single operational amplifier

- All load resistances identical

Glenn Research Center 


\section{Digital Hybrid}

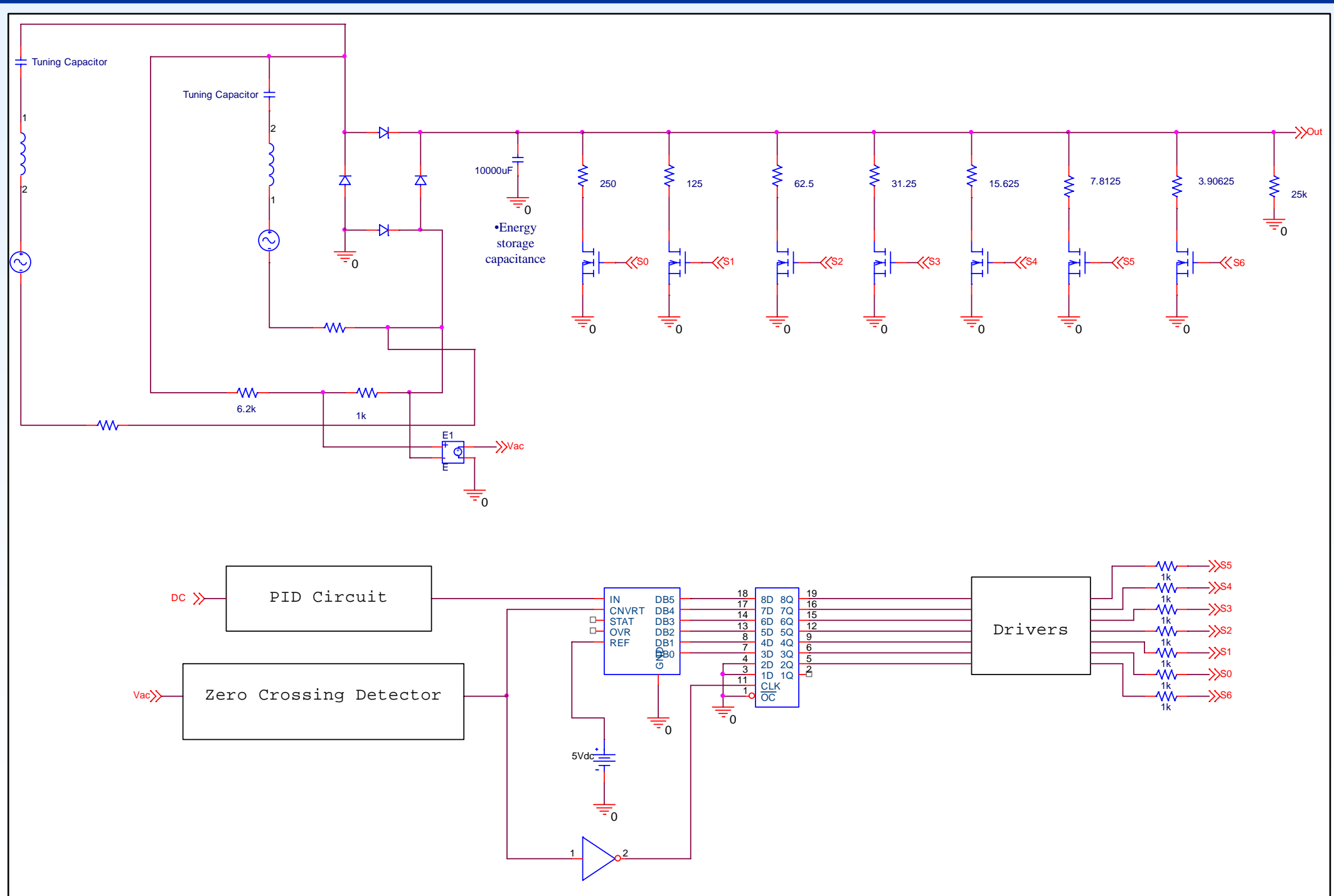

Glenn Research Center

at Lewis Field 


\section{Buck Circuit with Zener-diode}

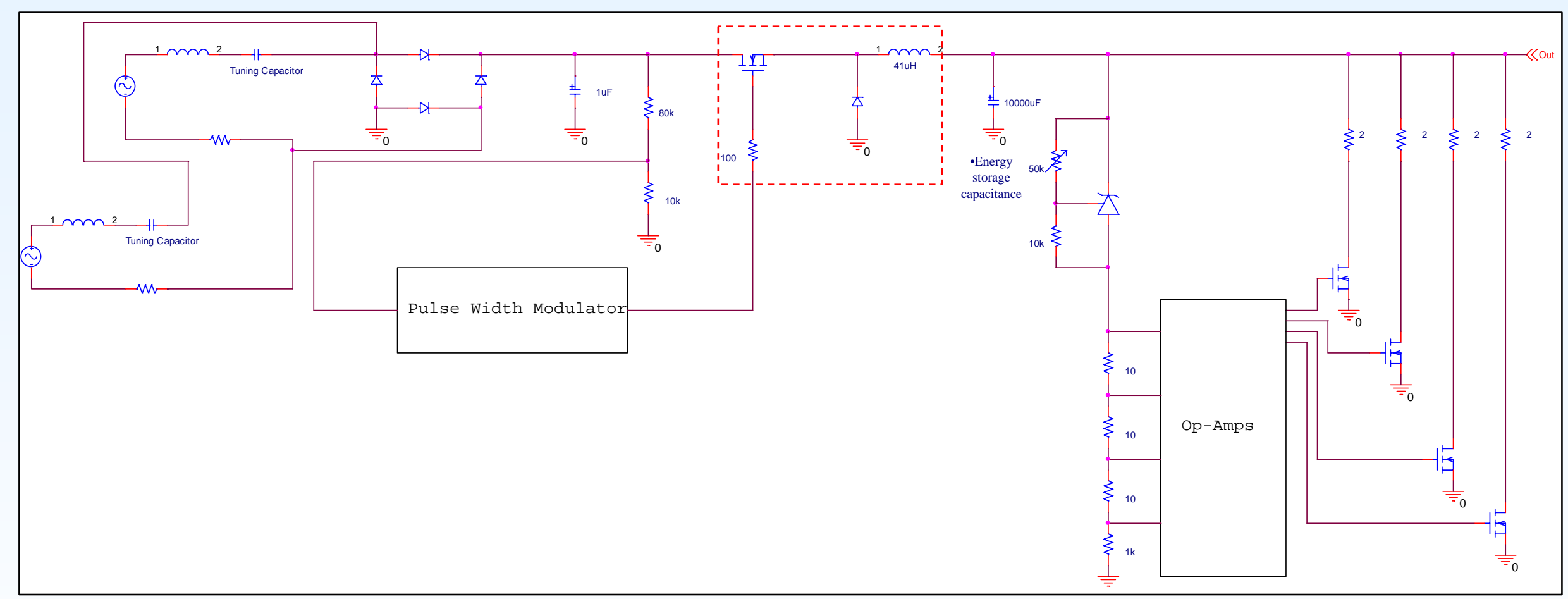

\section{Key Features}

- Reduces output voltage by one half - requires DC-DC converter

- Allows adjustment of load at frequency independent of convertor cycle 


\section{Boost Circuit with Pulse Width Modulator}

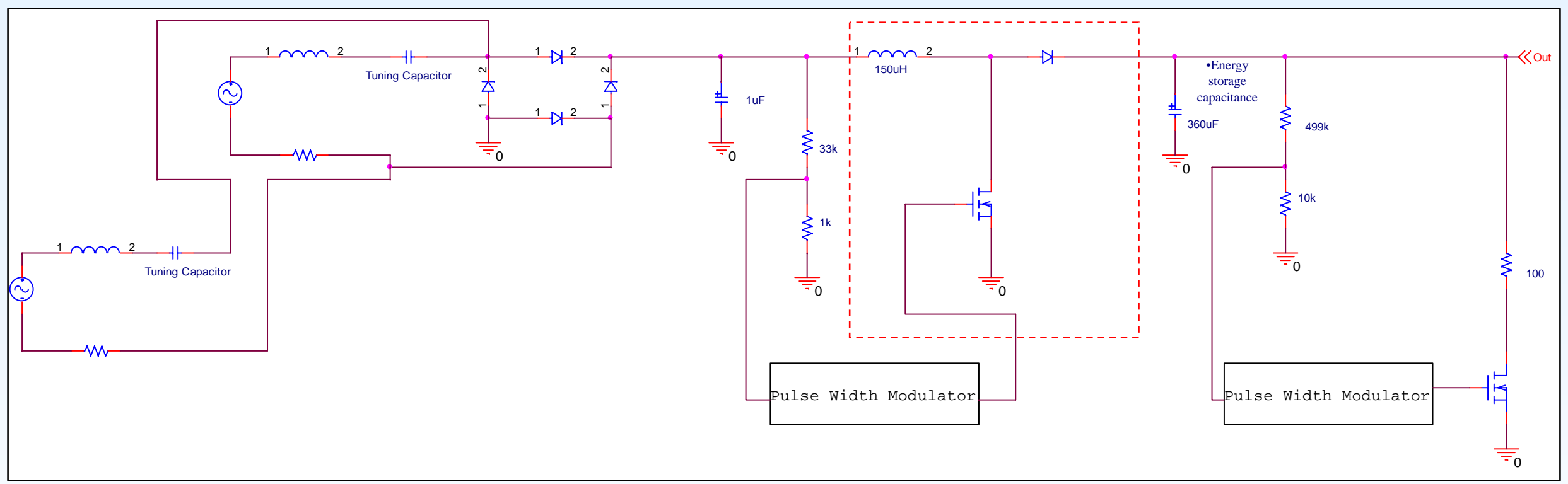

Key Features

- Doubles output voltage - requires DC-DC converter

- Allows load adjustment at frequency independent of convertor cycle

Glenn Research Center 


\section{Linear AC Regulator}

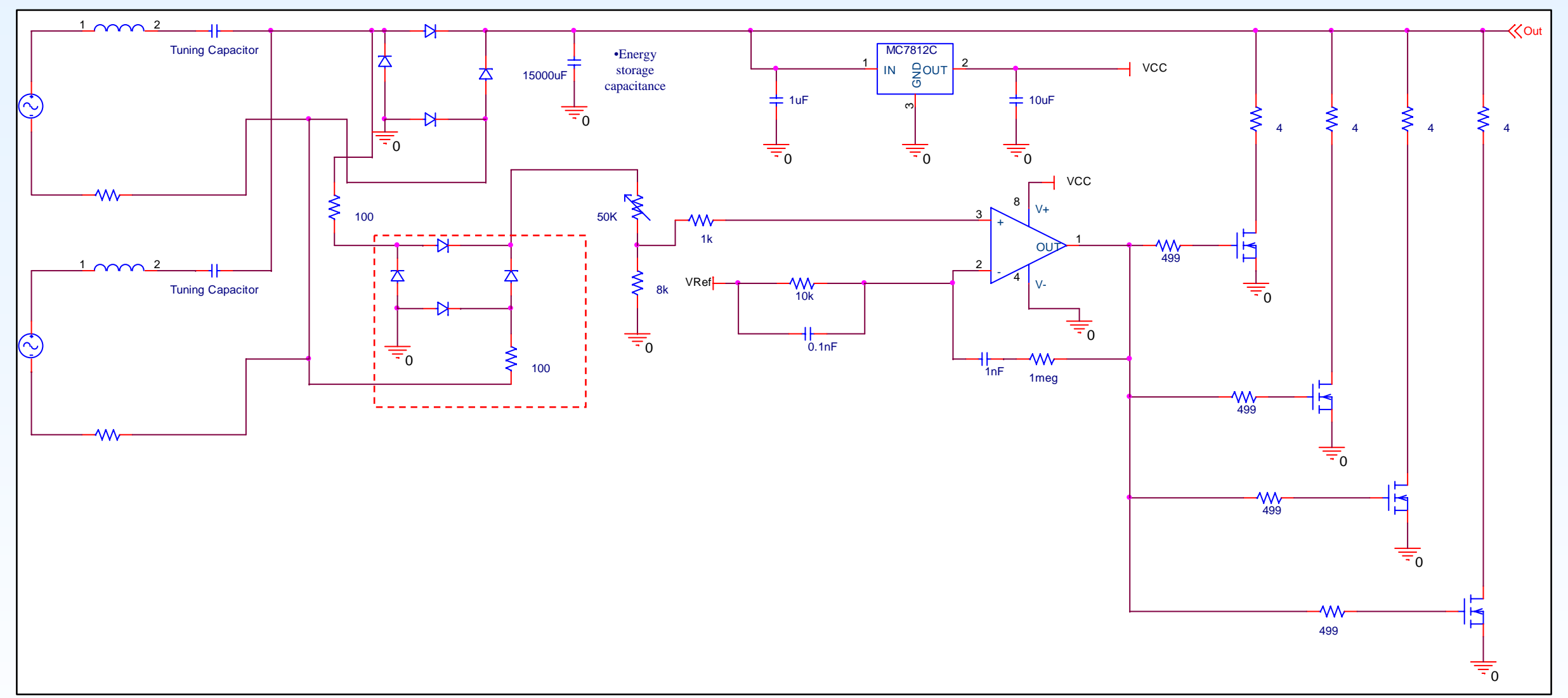

\section{Key Features}

- FETs driven in linear range - also dissipate power

- Load controlled by AC voltage sensing

- All load resistances identical

Glenn Research Center 


\section{Initial Option Elimination}

- $\quad$ Load Control - AC bus monitoring allows more accurate control of the load

- $\quad$ Load Stages - Increases reliability by adding redundancy. Controller does not fail if one load loses connectivity

- Power Dissipation - Resistors vs. Transistors (FETs). Transistors must be derated to dissipate power

- DC-DC Converter - Needed in some options to return to $28 \mathrm{~V}$ output. Contributes relatively large mass and volume

- $\quad$ Tuning - Process of customizing controller for specific Stirling convertor design.

\begin{tabular}{|c|c|c|c|}
\hline Controller option & Advantages & Disadvantages & Eliminated \\
\hline Zener-diode (baseline) & $\begin{array}{c}\text { Resistor power dissipation } \\
\text { Load staging }\end{array}$ & DC voltage monitoring & $\mathrm{X}$ \\
\hline Linear DC regulator & Load staging & $\begin{array}{c}\text { Transistor power dissipation } \\
\text { DC voltage monitoring }\end{array}$ & $\mathrm{X}$ \\
\hline Digital hybrid & Resistor power dissipation & $\begin{array}{c}\text { Transistor power dissipation } \\
\text { DC voltage monitoring } \\
\text { DC-DC converter required }\end{array}$ & $\mathrm{X}$ \\
\hline Buck circuit with zener-diode & $\begin{array}{c}\text { Resistor power dissipation } \\
\text { AC voltage monitoring } \\
\text { Load staging }\end{array}$ & $\begin{array}{c}\text { DC-DC converter required } \\
\text { Complex tuning }\end{array}$ & \\
\hline Boost circuit with PWM & AC voltage monitoring & $\begin{array}{c}\text { DC-DC converter required } \\
\text { Complex tuning } \\
\text { Lack of modeling capability }\end{array}$ & \\
\hline Linear AC regulator & AC voltage monitoring \\
Load staging & Transistor power dissipation & \\
\hline
\end{tabular}

Glenn Research Center 


\section{Further Evaluation}

- Stability - Capability to maintain output voltage and piston amplitude when operating conditions change

- Parts Count - Reducing number of components reduces troubleshooting complexity, increases reliability, and affects size

- Sensitivity - Effect of variation within component manufacturing tolerances

- Efficiency - Calculated using PSpice ${ }^{\mathrm{TM}}$ model. Majority of losses occur in rectifier bridge

\begin{tabular}{|c|c|c|c|c|}
\hline & $\begin{array}{c}\text { Zener-diode } \\
\text { (baseline) }\end{array}$ & $\begin{array}{c}\text { Linear AC } \\
\text { regulator }\end{array}$ & $\begin{array}{c}\text { Digital } \\
\text { hybrid }\end{array}$ & $\begin{array}{c}\text { Buck converter } \\
\text { with zener-diode }\end{array}$ \\
\hline DC ripple (V) & 0.3 & 0.08 & 0.5 & 0.4 \\
\hline DC overshoot (V) & 1 & 1 & 11.2 & 0 \\
\hline Number of components & 46 & 42 & 56 & 62 \\
\hline Heat sinks required & 4 & 4 & 4 & 5 \\
\hline Sensitivity & None & None & $\begin{array}{c}\text { Load } \\
\text { resistance }\end{array}$ & $\begin{array}{c}\text { Energy storage } \\
\text { capacitance }\end{array}$ \\
\hline Efficiency (\%) & 85.1 & 95.4 & 93.4 & 84.7 \\
\hline
\end{tabular}

Final Selection : Linear AC Regulator 


\section{Linear AC Regulator Testing}

- August 2006 : Buildup complete

- August 2006 : Checkout test

- $88 \mathrm{~W}_{\mathrm{e}}$ supplied by AC source with series resistor to simulate alternator coil resistance.

- Measured voltage waveforms. Agreed well with model predictions

- September 2006 : Operation with pair of EE-35 convertors up to $60 \mathrm{~W}_{\mathrm{e}}$

- December 2006 : Integrated into ASRG support electronics

- Operation successful up to maximum power of $130 \mathrm{~W}_{\mathrm{e}}$

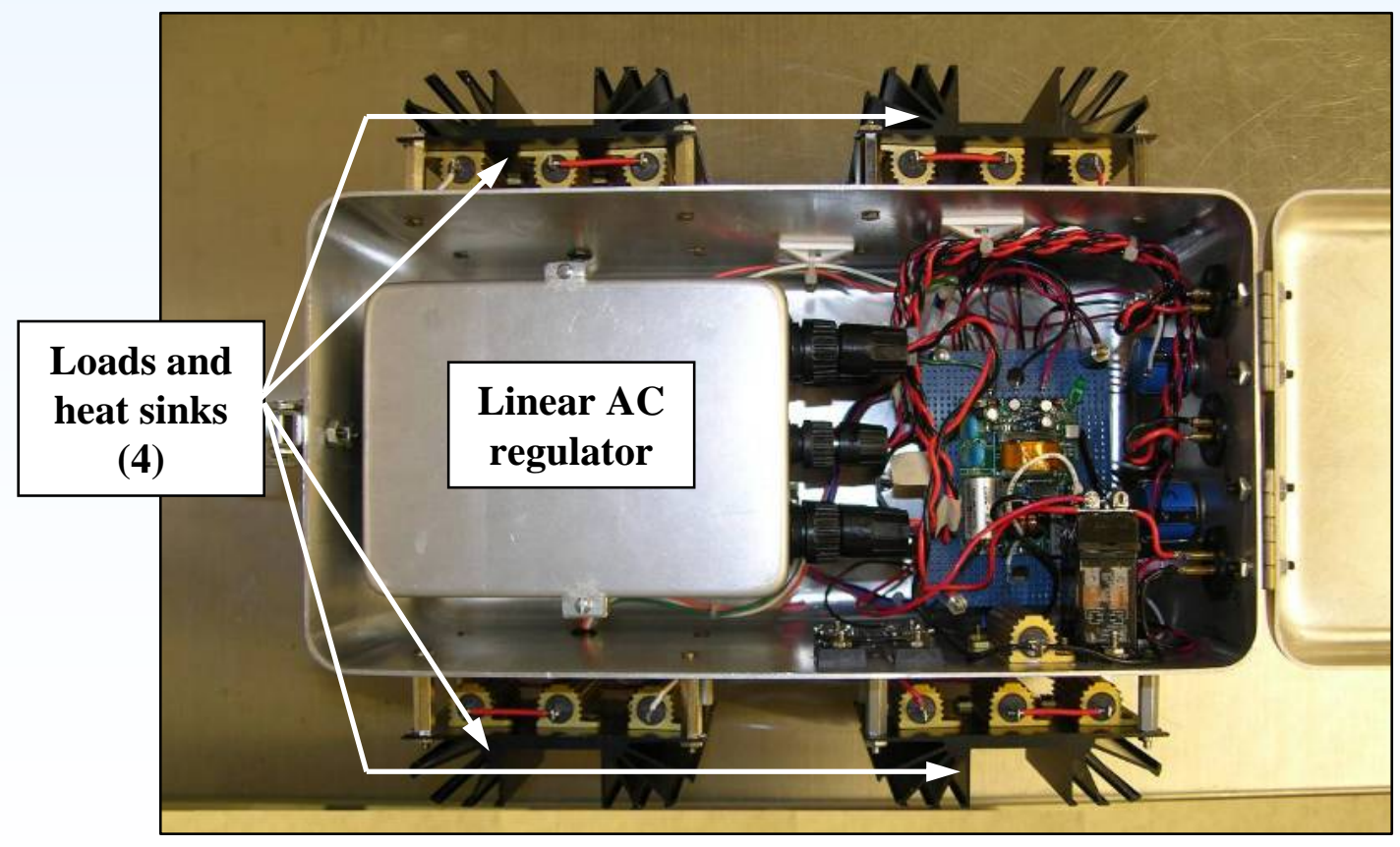

Glenn Research Center 


\section{ASC Testing}

Accomplishments

\section{ASC-0 \#1 and \#2}

- Over 2,225 hours of extended operation in both air and thermal vacuum environments

- Over 1,425 hours of operation at maximum hot-end temperature of $650{ }^{\circ} \mathrm{C}$

- Post processing performance data

\section{FTB Convertors \#5 and \#6}

- Successfully integrated for use in mobile power source for ASRG simulation

- Operated on novel, linear AC regulator controller up to full power of $130 \mathrm{~W}_{\mathrm{e}}$

Future Plans

- Summer 2007 :

- Receive ASC-1HS convertor pair

- Initiate extended operation in thermal vacuum at $850{ }^{\circ} \mathrm{C}$ hot-end

- Continue operation of ASC-0 pair in air

- Finalize ASRG Simulator and demonstrate use on rover

Glenn Research Center 